\title{
MULTIPLE CHOICES IMPLY THE INGLETON AND KREIN-MILMAN AXIOMS
}

\author{
MARIANNE MORILLON
}

\begin{abstract}
In set theory without the Axiom of Choice, we consider Ingleton's axiom which is the counterpart in ultrametric analysis of the Hahn-Banach axiom. We show that in ZFA, set theory without the Axiom of Choice weakened to allow "atoms", Ingleton's axiom does not imply the Axiom of Choice (this solves in ZFA a question raised by van Rooij, [20]). We also prove that in ZFA, the "multiple Choice" axiom implies the Krein-Milman axiom. We deduce that, in ZFA, the conjunction of the Hahn-Banach, Ingleton and Krein-Milman axioms does not imply the Axiom of Choice.
\end{abstract}

\section{INTRODUCTION}

We denote by ZFA (see [10, p. 44]) the set theory ZF with the axiom of extensionality weakened to allow the existence of atoms. We denote by MC ("Multiple Choice") the following consequence of the Axiom of Choice AC (see [10, p. 133] and form 67 of [7, p. 35]): "For every infinite family $\left(X_{i}\right)_{i \in I}$ of nonempty sets, there exists a family $\left(F_{i}\right)_{i \in I}$ of nonempty finite sets such that for each $i \in I, F_{i} \subseteq X_{i}$."

It is known that $\mathbf{M C}$ is equivalent to $\mathbf{A C}$ in $\mathbf{Z F}$ (see [10, Theorem 9.1 p. 133]), but $\mathbf{M C}$ does not imply AC in ZFA (see [10, Theorem 9.2 p. 134]). Given a prime number $p \geq 2$, we also consider the following refined statement for each prime number $p \geq 2$, where for every finite set $F$, we denote by $\# F$ the cardinal of $F$ :

$\operatorname{MC}(p)$ : "For every family $\left(A_{i}\right)_{i \in I}$ of nonempty sets, there exists a family $\left(B_{i}\right)_{i \in I}$ of finite sets such that for every $i \in I, B_{i} \subseteq A_{i}$ and $\# B_{i}$ is not a multiple of $p$."

The conjunction of all statements $\mathbf{M C}(p)$ for all prime numbers $p$ is denoted by form 218 in [7, p. 52]). Levy (1962) built a model $\mathcal{N} 6$ of ZFA (see [7, p. 185], 12]) satisfying $\mathbf{M C}(p)$ for every prime number $p \geq 2$ (and thus satisfying also $\mathbf{M C}$ ), in which there exists a sequence

Date: January 15, 2019.

2000 Mathematics Subject Classification. Primary 03E25 ; Secondary 46S10.

Key words and phrases. Axiom of Choice, non-Archimedean fields, Ingleton's theorem, Krein-Milman's theorem. 
$\left(F_{n}\right)_{n \in \mathbb{N}}$ of finite sets such that for every $n \in \mathbb{N}, \# F_{n}=n+1$ and $\prod_{n \in \mathbb{N}} F_{n}=\varnothing$ : such a model does not satisfy $\mathrm{AC}$ (and more precisely, this model does not satisfy the "countable Axiom of Choice for finite sets"). It follows that in ZFA, the conjunction of statements $\mathbf{M C}(p)$ for all prime numbers $p$ does not imply AC.

Consider the well-known Hahn-Banach statement HB (form 52 of [7):

HB: Given a real vector space $E$ and a sublinear functional $p: E \rightarrow \mathbb{R}$, there exists a linear functional $f: E \rightarrow \mathbb{R}$ such that $f \leq p$.

It is known (see [14, Corollary 1]) that in ZFA, MC implies Rado's selection Lemma RL (form 99 of [7]), and that RL implies HB (see [14, Theorem 1]), thus MC implies HB.

Given a spherically complete ultrametric valued field ( $\mathbb{F},|$.$| ) (see Sec-$ tion 2.5), Ingleton proved in set theory $(\mathbf{Z F}+\mathbf{A C})$ (see [9]), for each ultrametric normed space over a "spherically complete" ultrametric valued field ( $\mathbb{F},||$.$) , a "Hahn-Banach"-type result which we denote by \mathbf{I}_{(\mathbb{F},|.|)}$. A.C.M. van Rooij ([20]) showed that for each ultrametric valued field $(\mathbb{F},||$.$) such that the large ball \{x \in \mathbb{F}:|x| \leq 1\}$ of $\mathbb{F}$ is compact (whence $(\mathbb{F},||$.$) is spherically complete), BPI implies \mathbf{I}_{(\mathbb{F},|.|)}$. He asked whether the "full Ingleton theorem" (i.e. the conjunction of all statements $\mathbf{I}_{\mathbb{F},|.|}$ for all spherically complete ultrametric valued fields $(\mathbb{F},||)$.$) implies$ AC. We shall show that in set theory ZFA (set theory without choice weakened to allow "atoms"), the "full Ingleton theorem" does not imply AC. More precisely, for every prime number $p \geq 2, \mathbf{M C}(p)$ implies the statement $\mathbf{I}_{(\mathbb{F},|.|)}$ for each spherically complete ultrametric valued field $(\mathbb{F},||$.$) with null characteristic such that the restriction of the absolute$ value on $\mathbb{Q}$ is equivalent to the $p$-adic absolute value, and also for each spherically complete ultrametric valued field $(\mathbb{F},||$.$) with characteristic$ $p$ (see Corollary 2-(2) in Section 3). In ZFA, MC implies the statement $\mathbf{I}_{(\mathbb{F},|.|)}$ for each spherically complete ultrametric valued field ( $\left.\mathbb{F},||.\right)$ with null characteristic such that the restriction of the absolute value on $\mathbb{Q}$ is trivial (see Corollary 2t(1) in Section 3). Since Levy's above model of ZFA satisfies $\mathbf{M C}(p)$ for every prime number $p \geq 2$ (and thus it also satisfies MC), it follows that in ZFA, the "full Ingleton axiom" does not imply AC.

In Section 4, we prove (see Theorem 2) that in ZFA, MC implies the Krein-Milman statement (form 65 of [7]):

KM: Given a Hausdorff locally convex topological real vector space $E$, every nonempty compact convex subset of $E$ has an extreme point. We shall use "trees" of "facets" of a nonempty convex compact subset of a topological real vector space. We deduce that $\mathbf{M C} \Rightarrow(\mathbf{H B}+\mathbf{K M})$. 
This generalizes Pincus's following result ([17]): the model $\mathcal{N}_{2}$ (described in [7, p. 178]) of $\mathbf{Z F A}+\neg \mathbf{A C}+\mathbf{M C}$ satisfies $(\mathbf{K M}+\mathbf{H B})$. It follows that the conjunction of statements $\mathbf{M C}(p)$ for every prime number $p \geq 2$ implies Rado's lemma (and hence HB), the "full Ingleton axiom" and also the Krein-Milman axiom. In particular, Levy's model $\mathcal{N} 6$ of $\mathbf{Z F A}+\neg \mathbf{A C}$ satisfies all these statements.

The paper is organized as follows: in Section 2, we sketch basic results about ultrametric valued fields, in Section 3 we prove in ZFA that the conjunction of the statements $\mathbf{M C}(p)$ for all prime numbers $p$ implies the "full Ingleton Theorem", and in the last Section 4, we prove in ZFA that MC implies the "Krein-Milman" statement.

\section{Preliminaries on Ultrametric VAlUed FieldS}

2.1. Valued fields. An absolute value on a (commutative) field $\mathbb{F}$ is a mapping $||:. \mathbb{F} \rightarrow \mathbb{R}_{+}$satisfying the following three properties for every $\lambda, \mu \in \mathbb{F}:|\lambda|=0 \Leftrightarrow \lambda=0 ;|\lambda \mu|=|\lambda||\mu|$ and $|\lambda+\mu| \leq|\lambda|+|\mu|$. Each valued field $(\mathbb{F},||$.$) gives rise to a metric d_{|.|}: \mathbb{F} \times \mathbb{F} \rightarrow \mathbb{R}_{+}$defined by $d(x, y)=|x-y|$ for every $x, y \in \mathbb{F}$. An absolute value $|$.$| on \mathbb{F}$ is said to be ultrametric if the associated metric $d_{|\cdot|}$ is ultrametric (i.e. $\forall x, y, z \in \mathbb{F} d(x, z) \leq \max (d(x, y), d(y, z)))$, equivalently if for every $\lambda$, $\mu \in \mathbb{F},|\lambda+\mu| \leq \max (|\lambda|,|\mu|)$.

Example 1 (The trivial absolute value on a field). For each commutative field $\mathbb{F}$, the mapping $|\cdot|_{\text {triv }}: \mathbb{F} \rightarrow \mathbb{R}_{+}$associating to each $\lambda \in \mathbb{F}$ the real number 0 if $\lambda=0$ and 1 otherwise is an absolute value, called the trivial absolute value on $\mathbb{F}$. The associated metric is the discrete distance $d_{\text {disc }}$ on $\mathbb{F}$, satisfying $d_{d i s c}(x, y)=0$ if $x=y$ and 1 otherwise.

Remark 1. Notice that if $|$.$| is an absolute value on a field \mathbb{F}$, then $\left|1_{\mathbb{F}}\right|=1$. If $\mathbb{F}$ is a finite field with $m$ elements, then for every $x \in \mathbb{F} \backslash\{0\}$, $x^{m-1}=1$ thus $|x|^{m-1}=1$ so $|x|=1$; it follows that the trivial absolute value is the only absolute value on $\mathbb{F}$.

2.2. Ultrametric valued fields. Given a non-empty set $X$ and a semi-metric $d: X \times X \rightarrow \mathbb{R}_{+}$, a large ball of the semi-metric space $(X, d)$ is a ball with "large inequalities" i.e. a subset of $X$ of the form $B(a, r):=\{x \in X: d(x, a) \leq r\}$ where $a \in X$ and $r \in \mathbb{R}_{+}$. Given a ultrametric valued field $(\mathbb{F},||$.$) , the large ball B_{\mathbb{F}}(0,1)$ is a subring of $\mathbb{F}$, and the "strict ball" $\mathfrak{M}_{\mathbb{F}}:=\{x \in \mathbb{F}:|x|<1\}$ is the unique maximal ideal of the ring $B_{\mathbb{F}}(0,1)\left(\mathfrak{M}_{\mathbb{F}}\right.$ is the set of non-invertible elements of the ring $\left.B_{\mathbb{F}}(0,1)\right)$. The field $B_{\mathbb{F}}(0,1) / \mathfrak{M}_{\mathbb{F}}$ is the residue class field of the valued field $\mathbb{F}$. The mapping $|\cdot|_{\mid \mathbb{F} \backslash\{0\}}: \mathbb{F} \backslash\{0\} \rightarrow \mathbb{R}_{+}^{*}$ is a group morphism w.r.t. the multiplicative laws, thus the set $V_{(\mathbb{F},|.|)}:=\{|x|$ : 
$x \in \mathbb{F} \backslash\{0\}\}$ is a subgroup of the multiplicative group $\mathbb{R}_{+}^{*}$, which is called the value group of the absolute value |.|. The value group $V_{(\mathbb{F},|.|)}$ of a ultrametric absolute value |.| on a valued field $\mathbb{F}$ is either discrete or dense in $\mathbb{R}_{+}^{*}$.

Example 2 (The trivial absolute value on a field). For each commutative field $\mathbb{F}$ endowed with the trivial absolute value $|\cdot|_{\text {triv }}: \mathbb{F} \rightarrow \mathbb{R}_{+}$, then $B_{\mathbb{F}}(0,1)=\mathbb{F}$, and the maximal ideal $\mathfrak{M}_{\mathbb{F}}$ is the null ideal $\{0\}$, thus the residue class field of $\mathbb{F}$ is $\mathbb{F}$. The group of values $V_{\mathbb{F}}$ is the trivial subgroup $\{1\}$ of $\mathbb{R}_{+}^{*}$.

Example 3. Given a ultrametric valued field $(\mathbb{F},||$.$) such that V_{(\mathbb{F},|.|)}$ is discrete and $V_{\mathbb{F}} \neq\{1\}$, then $\left.s:=\max \left\{|x|: x \in \mathfrak{M}_{\mathbb{F}}\right\} \in\right] 0,1[$, and $V_{(\mathbb{F},|.|)}=\left\{s^{n}: n \in \mathbb{Z}\right\}$.

2.3. Cauchy-complete semi-metric spaces. Given a semi-metric space $(X, d)$, a filter $\mathcal{F}$ on $X$ is Cauchy if for every real number $\varepsilon>0$, there exists $F \in \mathcal{F}$ such that the d-diameter $\sup _{x, y \in F} d(x, y)$ of $F$ is $<\varepsilon$. A semi-metric space $(X, d)$ is Cauchy complete if every Cauchy filter of this semi-metric space has a non-empty intersection.

Example 4. (1) The field $\mathbb{R}$ of real numbers endowed with the usual absolute value is Cauchy complete. For every set $X$, denoting by $B(X, \mathbb{R})$ the vector space of bounded functions $f: X \rightarrow \mathbb{R}$, then the normed vector space $B(X, \mathbb{R})$ endowed with the uniform norm is Cauchy-complete.

(2) Every set $X$ endowed with the discrete distance $d_{\text {disc }}$ is Cauchycomplete since, for every real number $\varepsilon \in] 0,1[$, non-empty $\varepsilon$ small subsets of $X$ are singletons. In particular, every valued field endowed with the trivial absolute value is Cauchycomplete.

Remark 2. Say that a semi-metric space $(X, d)$ is sequentially complete if every $d$-Cauchy sequence of $X$ converges to a point of $X$. There are models of ZF (for example the "basic Cohen model" described in [10, Section 5.3]) in which there exists a dense (hence infinite) subset $D$ of $\mathbb{R}$ which is Dedekind-finite (i.e. which does not contain any subset equipotent with $\mathbb{N}$ ). Such a set $D$ endowed with the usual distance of $\mathbb{R}$ is a metric space which is "sequentially complete" (because for each sequence $\left(x_{n}\right)_{n \in \mathbb{N}}$ of $D$, the set $\left\{x_{n}: n \in \mathbb{N}\right\}$ is finite thus each Cauchysequence of $(D, d)$ is stationnary) but the metric subspace $(D, d)$ of $\mathbb{R}$ is not Cauchy-complete since $D$ is not closed in $\mathbb{R}$. 
A Cauchy-completion of a semi-metric space $(X, d)$ is a Cauchy complete metric space $(\hat{X}, \hat{d})$ together with an isometry $j:(X, d) \rightarrow(\hat{X}, \hat{d})$ such that $j[X]$ is dense in $\hat{X}$.

Proposition 1. For every semi-metric space $(X, d)$, the mapping $j$ : $X \rightarrow B(X, \mathbb{R})$ associating to each $a \in X$ the mapping $f_{a}=(d(x, a))_{x \in X}$ is isometric, the closed subspace $\overline{j[X]}$ of $B(X, \mathbb{R})$ is Cauchy-complete, and $(j, \overline{j[X]})$ is a Cauchy-completion of $(X, d)$.

Given a valued field $(\mathbb{F},||$.$) , the laws +$ and . of the field $\mathbb{F}$ are uniquely extendable into continuous laws on the Cauchy-completion $\hat{\mathbb{F}}$ of the metric space $\left(\mathbb{F}, d_{|\cdot|}\right)$ (the law + is uniformly continuous and the law . is uniformly continuous on $\left.B_{\mathbb{F}}(0,1) \times B_{\mathbb{F}}(0,1)\right)$. The structure $\hat{\mathbb{F}}$ endowed with these extended laws is a valued field.

\subsection{Non trivial absolute values on $\mathbb{Q}$.}

2.4.1. Archimedean absolute values on $\mathbb{Q}$. An absolute value on a field $\mathbb{F}$ is archimedean if $\sup \left\{\left|n .1_{\mathbb{F}}\right|: n \in \mathbb{N}\right\}=+\infty$. Given a field $\mathbb{F}$, an absolute value on $\mathbb{F}$ is non archimedean if and only if it is ultrametric (see [19, Theorem 1.1 p. 2]).

Example 5. (1) The mapping $|\cdot|_{\mathbb{Q}}: \mathbb{Q} \rightarrow \mathbb{R}_{+}$associating to each rational number $x$ le number $\max (x,-x)$ is an archimedean absolute value on the field $\mathbb{Q}$ of rational numbers.

(2) Denoting by $\mathbb{R}$ the ordered field of real numbers, i.e. the Cauchy-completion of the valued field $(\mathbb{Q},|\cdot| \mathbb{Q})$, the "usual" absolute value $|\cdot|_{\mathbb{R}}: \mathbb{R} \rightarrow \mathbb{R}_{+}$which associates to each $x \in \mathbb{R}$ the real number $\max (x,-x)$ is archimedean.

(3) Denoting by $\mathbb{C}$ the field of complex numbers, the "modulus function" $|\cdot|_{\mathbb{C}}: \mathbb{C} \rightarrow \mathbb{R}_{+}$is an archimedean absolute value on $\mathbb{C}$.

(4) Given any subfield $\mathbb{F}$ of $\mathbb{C}$, the restriction of the "modulus function" $|\cdot|_{\mathbb{F}}:=|\cdot|_{\mathbb{C}_{\mathbb{F}}}: \mathbb{F} \rightarrow \mathbb{R}_{+}$is an archimedean absolute value on $\mathbb{F}$.

Remark 3. Notice that, given a subfield $\mathbb{F}$ of $\mathbb{C}$, for each real number $\tau \in] 0,1]$, the function $|\cdot|_{\mathbb{C}}^{\tau}{ }_{\mathbb{F}}$ is also an archimedean absolute value on $\mathbb{F}$. Given a ultrametric field $(\mathbb{F},||$.$) , for each real number \tau>0$, the function $|.|^{\tau}$ is also a ultrametric absolute value on $\mathbb{F}$ which is equivalent to the initial absolute value $|$.$| .$

2.4.2. p-adic absolute values on $\mathbb{Q}$. We denote by PRIME the set of prime natural numbers. For each $p \in \mathrm{PRIME}$, we denote by $v_{p}$ : $\mathbb{Q} \rightarrow \mathbb{Z} \cup\{+\infty\}$ the $p$-adic valuation on $\mathbb{Q}$, such that $v_{p}(0)=+\infty$, $v_{p}(1)=v_{p}(-1)=0$, and for all $x \in \mathbb{Q} \backslash\{0,1,-1\}, v_{p}(x)$ is the exponent 
$\alpha$ of $p$ in the prime decomposition $x= \pm p^{\alpha} \prod_{q \in \text { PRIME } ; q \neq p} q^{\alpha_{q}}$ of $x$ in prime numbers. For each prime natural number $p$, we denote by $\mathbb{F}_{p}=$ $\{0,1, \ldots, p-1\}$ the finite field with $p$ elements.

Proposition 2 ([5, Prop. 2.4 .3 p. 40]). (1) The mapping $x \mapsto|x|_{p}:=$ $p^{-v_{p}(x)}$ is a ultrametric absolute value on the field $\mathbb{Q}$ of rational numbers.

(2) The group of values of of the valued field $\left(\mathbb{Q},|\cdot|_{p}\right)$ is $\left\{p^{n}: n \in \mathbb{Z}\right\}$;

(3) The subring $B(0,1)$ of $\left(\mathbb{Q},|\cdot|_{p}\right)$ is the subset $\left\{\frac{m}{n}: m \in \mathbb{Z} ; n \in\right.$ $\mathbb{N}^{*}$ s.t. $\left.p \nmid n\right\}$ and thus $\mathbb{Z}$ is a subring of $B(0,1)$.

(4) The maximal ideal $\mathfrak{M}_{\left(\mathbb{Q},\left.|\cdot|\right|_{p}\right)}$ is $p B(0,1)$.

(5) The cosets of the ring $B(0,1)$ modulo the maximal ideal $p B(0,1)$ are the sets $i+p B(0,1)$ where $i \in\{0, \ldots, p-1\}$, and the residue class field is the finite field $\mathbb{F}_{p}$.

Proof. (11) and (2) are trivial. See the proofs of (3) and (44) in [5, Prop. 2.4.3-(i) and (ii) p. 40]. (5) We first notice that if $i, j \in\{0, \ldots, p-$ $1\}$, then the sum of the two classes $i+p B(0,1)$ and $j+p B(0,1)$ is the class $r+p B(0,1)$ where $r$ is the remainder in the euclidean division of $(i+j)$ by $p$ : indeed, let $q \in \mathbb{N}$ such that $i+j=q p+r$ where $r \in\{0, \ldots, p-1\}$; then $|i+j-r|_{p}=|q p|_{p}$ which is 0 if $q=0$ and $p^{-\alpha}$ where $\alpha \in \mathbb{N}^{*}$ else, thus $|i+j-r|_{p}<1$ so $(i+j)+p B(0,1)=$ $r+p B(0,1)$. Let $x \in \mathbb{Q}$ such that $|x|_{p}=1$. Let us show that there exists $i \in\{1, \ldots, p-1\}$ such that $|x-i|_{p}<1$. It is sufficient to prove this if $x=\frac{1}{n}$ where $n \in \mathbb{N}^{*}$ with $|n|_{p}=1$ i.e. $p$ does not divide $n$. Since $p \nmid n, n+p \mathbb{Z}$ is invertible in $\mathbb{Z} / p \mathbb{Z}$, thus let $i \in\{1, \ldots, p-1\}$ such that $p /(n i-1)$; then $|n i-1|_{p}<1$ i.e. $|n|_{p}\left|i-\frac{1}{n}\right|_{p}<1$ i.e. $\left|i-\frac{1}{n}\right|_{p}<1$ thus $\frac{1}{n}-i \in p B(0,1)$.

Proposition 3 ([5, Lemma 3.2 .3 p. 51]). (1) The valued field $\left(\mathbb{Q},|\cdot|_{p}\right)$ is not complete.

(2) Denoting by $\mathbb{Q}_{p}$ the Cauchy-completion of the valued field $\left(\mathbb{Q},|\cdot|_{p}\right)$, the extended absolute value $|\cdot|_{p}: \mathbb{Q}_{p} \rightarrow \mathbb{R}_{+}$is also ultrametric and has the same group of values as $\left(\mathbb{Q},|\cdot|_{p}\right)$. Moreover, $p B_{\mathbb{Q}_{p}}(0,1)$ is still a maximal ideal of the subring $B_{\mathbb{Q}_{p}}(0,1)$ of $\mathbb{Q}_{p}$ and the quotient field $B_{\mathbb{Q}_{p}}(0,1) / p B_{\mathbb{Q}_{p}}(0,1)$ is still the finite field $\mathbb{F}_{p}$.

Theorem (Ostrowski's theorem, [19, Th. 1.2 p. 3]). Every non trivial absolute value on $\mathbb{Q}$ is of the form $|\cdot|_{\mathbb{R}}^{\tau}$ where $\tau$ is some real number such that $0<\tau<1$, or of the form $|\cdot|_{p}^{\tau}$ for some prime number $p$ and $\tau>0$. 
2.5. Spherically complete ultrametric spaces. A ultrametric semimetric space is spherically complete if every chain of large balls of this semi-metric space has a non-empty intersection.

Example 6. Each nonempty set $X$ endowed with the discrete metric $d_{d i s c}$ is spherically complete, because large balls of the metric space $\left(X, d_{d i s c}\right)$ are singletons of $X$ or the whole set $X$.

Proposition 4. Every spherically complete ultrametric space is Cauchycomplete.

Proof. Assume that $(X, d)$ is a ultrametric metric space which is spherically complete and that $\mathcal{F}$ is a Cauchy filter of the metric space $(X, d)$. Consider the set $\mathcal{C}$ of large balls which belong to $\mathcal{F}$. For every $B_{1}$, $B_{2} \in \mathcal{C}, B_{1} \cap B_{2} \in \mathcal{F}$ thus $B_{1} \cap B_{2} \neq \varnothing$; since $d$ is ultrametric, it follows that $B_{1} \subseteq B_{2}$ or $B_{2} \subseteq B_{1}$. Thus $\mathcal{C}$ is a chain of large balls. Notice that for every $F \in \mathcal{F}$ with diameter $d_{F}$, for every $x \in F$, $F \subseteq B\left(x, d_{F}\right)$, thus, for every $x, y \in F, B\left(x, d_{F}\right)=B\left(y, d_{F}\right)$. Since $(X, d)$ is spherically complete, the set $\cap \mathcal{C}$ is nonempty. Since $\mathcal{F}$ is a Cauchy-filter, $\mathcal{C}$ contains balls of diameter $\varepsilon$ for every $\varepsilon>0$, thus $\cap \mathcal{C}$ is a singleton $\{a\}$. We now show that for every $\varepsilon>0$ and every $F \in \mathcal{F}$, $B(a, \varepsilon) \cap F \neq \varnothing$ (whence it will follow that $a \in \bar{F}$ ). It is sufficient to prove that for every $\varepsilon>0$ and every $F \in \mathcal{F}$ such that $\operatorname{diam}(F)<\varepsilon / 2$, $B(a, \varepsilon) \cap F \neq \varnothing$. Given some $F \in \mathcal{F}$ such that $\operatorname{diam}(F)<\varepsilon / 2$, then, for every $x \in F, F \subseteq B(x, \varepsilon / 2)$, thus $B(x, \varepsilon / 2) \in \mathcal{C}$, so $a \in B(x, \varepsilon / 2)$ whence $B(a, \varepsilon / 2) \cap F \neq \varnothing$.

Remark 4. For each prime number $p$, the valued field $\left(\mathbb{Q},|\cdot|_{p}\right)$ is not complete hence not spherically complete,

\subsection{For each prime number $p, \mathbb{Q}_{p}$ is locally compact hence spherically complete.}

2.6.1. The topological ring $\mathbb{Z}_{p}$. Let $p$ be a prime number. For each natural number $n$, we endow the finite ring $\mathbb{Z} / p^{n} \mathbb{Z}$ with the discrete topology and we endow the product $\operatorname{ring} \mathcal{A}:=\prod_{n \in \mathbb{N}^{*}} \mathbb{Z} / p^{n} \mathbb{Z}$ with the product topology: thus the topological $\operatorname{ring} \mathcal{A}$ is compact and metrizable (by the usual metrics on the product of metric spaces $\mathcal{A}$ ). We denote by $\mathbb{Z}_{p}$ (see [1, Prop. 1.2.1 p. 18]) the closed subring $\left\{x=\left(x_{n}\right)_{n \in \mathbb{N}^{*}} \in \mathcal{A}\right.$ : $\left.\forall n \in \mathbb{N}^{*} p^{n} \mid\left(x_{n+1}-x_{n}\right)\right\}$ of $\mathcal{A}$. It follows that $\left(\mathbb{Z}_{p},+, \times\right)$ is a commutative compact topological ring. The topological subspace $\mathbb{Z}_{p}$ of $\mathcal{A}$ is also metrizable. The unit of $\mathbb{Z}_{p}$ is the constant sequence (1). Let can $: \mathbb{Z} \rightarrow \mathbb{Z}_{p}$ be the canonical injection. If $a \in \mathbb{N}$, if $a=\sum_{k} a_{k} p^{k}$ is the $p$-ary expansion of $a$ (where each $a_{k} \in\{0, \ldots, p-1\}$, then $\operatorname{can}(a)$ is the 
element $x=\left(x_{n}\right)_{n \in \mathbb{N}^{*}}$ of $\mathbb{Z}_{p}$ such that for each $n \geq 1, x_{n}=\sum_{0 \leq k<n} a_{k} p^{k}$. It follows that can $: \mathbb{Z} \rightarrow \mathbb{Z}_{p}$ is dense (see [1, Prop. 1.2 .3 p. 19].

Proposition 5 ([1, Prop. 1.4.5. p. 23]). An element $x=\left(x_{n}\right)_{n \in \mathbb{N}^{*}}$ is invertible in $\mathbb{Z}_{p}$ if and only if $x_{1} \neq 0$ in $\mathbb{Z} / p \mathbb{Z}$.

2.6.2. The topological ring $\mathbb{Z}_{p}$ and the large ball $B(0,1)$ of $\mathbb{Q}_{p}$ are isomorphic.

Proposition 6. The topological ring $\mathbb{Z}_{p}$ and the large unit ball $B(0,1)$ of $\mathbb{Q}_{p}$ are isomorphic. It follows that the large ball $B(0,1)$ of $\mathbb{Q}_{p}$ is compact.

Proof. For each $x=\left(x_{n}\right)_{n \in \mathbb{N}^{*}} \in \mathbb{Z}_{p}$, considering each $x_{n} \in \mathbb{Z} / p^{n} \mathbb{Z}$ as an element of $\left\{0, \ldots, p^{n}-1\right\} \subseteq \mathbb{Z}$, the sequence $\left(x_{n}\right)_{n \geq 1}$ of $\mathbb{Q}$ is a Cauchy sequence for the metric $d_{p}$ on $\mathbb{Q}$, thus it converges to a unique element of $B_{\mathbb{Q}_{p}}(0,1)$. Let $f: \mathbb{Z}_{p} \rightarrow B_{\mathbb{Q}_{p}}(0,1)$ be the mapping associating to each $x=\left(x_{n}\right)_{n \in \mathbb{N}^{*}} \in \mathbb{Z}_{p}$ its limit in $B_{\mathbb{Q}_{p}}(0,1)$. Then $f$ is a morphism of rings, and $f$ is one-to-one. Moreover, the mapping $f$ is onto: since $f\left[\mathbb{Z}_{p}\right]$ is a compact (hence closed) subset of $B(0,1)$, it is sufficient to prove that $\left(\mathbb{Q} \cap B_{\mathbb{Q}_{p}}(0,1)\right) \subseteq f\left[\mathbb{Z}_{p}\right]$, or, equivalently, that for each $n \in \mathbb{N}^{*}$ such that $p \nmid n, \frac{1}{n} \in f\left[\mathbb{Z}_{p}\right]$ : given $n \in \mathbb{N}^{*}$ such that $p \nmid n$, consider the $p$-ary expansion $n=\sum_{k} a_{k} p^{k}$ where each $a_{k} \in\{0, \ldots, p-1\}$, then $a_{0} \neq 0$ thus $\operatorname{can}(n)=\left(a_{0}, a_{0}+p a_{1}, \ldots\right)$ is invertible in $\mathbb{Z}_{p}$, so $\frac{1}{n} \in f\left[\mathbb{Z}_{p}\right]$.

Corollary 1. The Cauchy completion $\mathbb{Q}_{p}$ of $\left(\mathbb{Q},|\cdot|_{p}\right)$ is spherically complete.

Proof. Using the previous Proposition, every large ball of $\mathbb{Q}_{p}$ is compact whence the metric space $\left(\mathbb{Q},|\cdot|_{p}\right)$ is Cauchy-complete.

Remark 5. Since the field $\mathbb{Q}$ is countable, $\mathbb{Q}$ has a unique countable algebraic closure $\mathbb{Q}_{a c}$. For each prime number $p$, there exists a unique absolute value on $\mathbb{Q}_{a c}$ extending $|\cdot|_{p}$ (using [1, Prop. 2.6.1 p. 63]). The Cauchy-completion of the valued field $\left(\mathbb{Q}_{a c},|\cdot|_{p}\right)$ is still algebraically closed and is denoted by $\mathbb{C}_{p}$ : thus $\mathbb{C}_{p}$ is a separable complete valued field (so it contains $\mathbb{Q}_{p}$ ) which is algebraically closed (thus contains an algebraic closure of $\mathbb{Q}_{p}$ ). The valued field $\mathbb{C}_{p}$ is complete but not spherically complete (see [18, Section 3.4]), hence not locally compact. Notice that there are models of ZFA (see [11]) and of ZF (see [6]) in which $\mathbb{Q}$ has a (non well orderable hence non countable) algebraic closure $\mathbb{L}$ without any non trivial absolute value on $\mathbb{L}$.

2.7. Semi-normed vector spaces over a valued field. Given a vector space $E$ over a valued field $(\mathbb{F},||$.$) , a semi-norm on E$ is a mapping $N: E \rightarrow \mathbb{R}_{+}$satisfying for every $x, y \in E$ and $\lambda \in \mathbb{F}$ the 
properties $N(\lambda . x)=|\lambda| N(x)$ and $N(x+y) \leq N(x)+N(y)$. For a ultrametric valued field $(\mathbb{F},||$.$) , the semi-norm N$ is ultrametric if the semi-metric associated to $N$ is ultrametric, equivalently if for every $x, y \in E, N(x+y) \leq \max (N(x), N(y))$.

\section{IN ZFA, $\forall^{\mathrm{PRIME}} p \mathbf{M C}(p)$ IMPLIES THE WHOLE INGLETON STATEMENT}

\subsection{The Hahn-Banach axiom and the Ingleton axiom.}

3.1.1. AC implies the Hahn-Banach statement. The following statement is a consequence of $\mathrm{AC}$ :

HB: (Hahn-Banach statement) Given a $\mathbb{R}$-vector space E, a seminorm $N: E \rightarrow \mathbb{R}_{+}$, a vector subspace $V$ of $E$ and a linear form $f: V \rightarrow \mathbb{R}$ such that for every $x \in V,|f(x)|_{\mathbb{R}} \leq N(x)$, there exists a linear form $\tilde{f}: E \rightarrow \mathbb{R}$ extending $f$ such that for every $x \in E$, $|\tilde{f}(x)|_{\mathbb{R}} \leq N(x)$.

The usual proof of $\mathbf{H B}$ can be obtained by transfinitely iterating the following Lemma (for example using Zorn's lemma or a transfinite recursion on ordinals and the Axiom of Choice).

Lemma 1 (Hahn-Banach, 1932, "one step"). Let E be a $\mathbb{R}$-vector space, let $N: E \rightarrow \mathbb{R}_{+}$be a semi-norm on $E$, let $V$ be a vector subspace of $E$ and let $f: V \rightarrow \mathbb{R}$ be a linear form such that $|f|_{\mathbb{R}} \leq N_{\mid V}$. For every $a \in E \backslash V$, there exists a linear form $\tilde{f}: V+\mathbb{R} . a \rightarrow \mathbb{R}$ extending $f$ such that $|\tilde{f}|_{\mathbb{R}} \leq N_{\mid V+\mathbb{R} . a}$.

Remark 6. In set-theory without the axiom of choice ZFA:

(1) $\mathbf{A C} \Rightarrow \mathbf{B P I} \Rightarrow \mathbf{R L} \Rightarrow \mathbf{H B}$ : see Jech's book [10] or Howard and Rubin's book [7].

(2) The implications $\mathbf{A C} \Rightarrow \mathbf{B P I}$ and $\mathbf{R L} \Rightarrow \mathbf{H B}$ are not reversible in $\mathbf{Z F}$ : see [10] or [7] for the first implication and [14, Remark 9] for the second one. It is known that RL does not imply BPI in ZFA ([8]) but the question "Does RL imply BPI?" is open in $\mathbf{Z F}$.

(3) $\mathbf{H B} \Rightarrow$ "The Hausdorff-Banach-Tarski" paradox (see [16]). In set theory ZFA, this implication is not reversible since the statement " $\mathbb{R}$ is well orderable" implies the Hausdorff-Banach-Tarski paradox but does not imply $\mathbf{H B}$.

3.1.2. AC implies Ingleton's statement. The following "one-step" result is the ultrametric counterpart of Lemma 1 . 
Lemma 2 (Ingleton, 1952, "one step"). Let E be a vector space over a spherically complete ultrametric valued field $(\mathbb{F},||$.$) , let N: E \rightarrow \mathbb{R}_{+}$ be a ultrametric semi-norm, let $V$ be a vector subspace of $E$ and let $f: V \rightarrow \mathbb{F}$ be a linear form such that $|f| \leq N_{\mid V}$. If $a \in E \backslash V$, then there exists a linear form $\tilde{f}: V+\mathbb{F} . a \rightarrow \mathbb{F}$ extending $f$ such that $|\tilde{f}| \leq N_{\uparrow V+\mathbb{F} . a}$.

Remark 7. Notice that both one-step Lemmas 1 and 2 extend to a "finite number of steps".

For each spherically complete ultrametric valued field $(\mathbb{F},||$.$) , the$ Axiom of Choice implies the following statement (see [9]):

$\mathbf{I}_{(\mathbb{F},|.|)}$ (Ingleton's statement): "Let $E$ be a $\mathbb{F}$-vector space, let $N: E \rightarrow$ $\mathbb{R}_{+}$be a ultrametric semi-norm, let $V$ be a vector subspace of $E$ and let $f: V \rightarrow \mathbb{F}$ be a linear form such that $|f| \leq N_{\uparrow V}$. Then there exists a linear form $\tilde{f}: E \rightarrow \mathbb{F}$ extending $f$ such that $|\tilde{f}| \leq N$."

We shall show that in set theory ZFA, the "full Ingleton theorem" + HB does not imply AC (unless ZFA is inconsistent). This answers in ZFA a question raised by van Rooij (see [20]).

3.2. A model of ZFA $+\neg \mathbf{A C}$ with "multiple choices". Levy (1962) built a model $\mathcal{N} 6$ of ZFA (see [7, p. 185], [12]) in which there exists a sequence $\left(F_{n}\right)_{n \in \mathbb{N}}$ of finite sets such that for every $n \in \mathbb{N}, \# F_{n}=n+1$ and $\prod_{n \in \mathbb{N}} F_{n}=\varnothing$ : such a model does not satisfy AC. However, Levy showed that this model satisfies the following "Multiple Choice" axiom: MC: ("Multiple Choice") "For every family $\left(A_{i}\right)_{i \in I}$ of non-empty sets, there exists a family $\left(B_{i}\right)_{i \in I}$ of non-empty finite sets such that for every $i \in I, B_{i} \subseteq A_{i}$."

and also the following refined statement for each prime number $p \geq 2$ : $\mathbf{M C}(p)$ : "For every family $\left(A_{i}\right)_{i \in I}$ of nonempty sets, there exists a family $\left(B_{i}\right)_{i \in I}$ of finite sets such that for every $i \in I, B_{i} \subseteq A_{i}$ and $\# B_{i}$ is not a multiple of $p . "$

Remark 8. In set-theory ZFA, $\mathbf{A C} \Rightarrow \forall^{\mathrm{PRIME}} p \mathbf{M C}(p) \Rightarrow \mathbf{M C}$ and none of these implications is reversible. In set-theory $\mathbf{Z F}$ (without atoms), MC implies AC.

3.3. $\forall^{\mathrm{PRIME}} p \mathrm{MC}(p)$ implies the "Full Ingleton" statement. Given a valued field $(\mathbb{F},||$.$) , a \mathbb{F}$-vector space $E$ endowed with a $\mathbb{F}$-seminorm $N$, a finite linear extender on $E$ is a mapping associating to each ordered pair $(V, f)$ where $V$ is a proper vector subspace of $E$ and $f: V \rightarrow \mathbb{F}$ is a linear form such that $|f| \leq N_{\uparrow V}$, an ordered pair $\left(V^{\prime}, f^{\prime}\right)$ such that $V^{\prime}$ is a vector subspace of $E$ strictly including $V$ such that the $\mathbb{F}$-vector space $V^{\prime} / V$ is finitely generated, and a linear mapping 
$f^{\prime}: V^{\prime} \rightarrow \mathbb{F}$ extending $f$ with $\left|f^{\prime}\right| \leq N_{\uparrow V^{\prime}}$. We shall prove in ZFA the following result:

Theorem 1. Let $(\mathbb{F},||$.$) be a spherically complete ultrametric valued$ field or the usual valued field $\mathbb{R}$. Let $E$ be a $\mathbb{F}$-vector space endowed with a semi-norm $N$ which is assumed to be ultrametric if $\mathbb{F} \neq \mathbb{R}$.

(1) If $\mathbb{F}$ is the archimedean field $\mathbb{R}$ or if $\mathbb{F}$ has characteristic zero and if $|\cdot|_{\mathbb{Q}}$ is the trivial absolute value, then $\mathbf{M C}$ implies a finite linear extender on $E$.

(2) In the other cases, there exists a prime number $p$ such that the field $\mathbb{F}$ has characteristic $p$ or such that the ultrametric valued field $(\mathbb{F},||$.$) has characteristic zero and |\cdot|_{\mathbb{Q}}$ is equivalent to $|\cdot|_{p}$; in these cases, $\mathbf{M C}(p)$ implies a finite linear extender on $E$.

Proof. (1) With MC, let $\Phi$ be a mapping associating to each non-empty subset $X$ of $E \cup \mathbb{F}^{E}$ a finite non-empty subset of $X$. Given a proper vector subspace $V$ of $E$ and a linear form $f: V \rightarrow \mathbb{F}$ satisfying $|f| \leq N_{\uparrow V}$, let $F:=\Phi(E \backslash V)$ and let $V_{F}:=\operatorname{span}(V \cup F)$. Using Hahn-Banach's one-step Lemma 1 (for $\mathbb{F}=\mathbb{R}$ ) or Ingleton's one-step Lemma 2 (otherwise), the set $\mathcal{G}$ of linear forms $g: V_{F} \rightarrow \mathbb{F}$ extending $f$ such that $|g| \leq N_{\uparrow V_{F}}$ is non-empty. Let $G:=\Phi(\mathcal{G})$. Consider the linear form $\tilde{f}:=$ $\frac{1}{\# G} \sum_{g \in G} g$ on $V_{F}$ : then $\tilde{f}$ extends $f$. If $\mathbb{F}=\mathbb{R}$, then $|\# G|_{\mathbb{R}}=\# G$ thus $|\tilde{f}(x)|_{\mathbb{R}}=\frac{1}{\# G}\left|\sum_{g \in G} g(x)\right|_{\mathbb{R}} \leq \frac{1}{\# G} \sum_{g \in G}|g(x)|_{\mathbb{R}} \leq N_{\uparrow V_{F}}(x)$. If $\mathbb{F}$ has characteristic zero and the restriction $\mid \cdot \|_{\mathbb{Q}}$ is the trivial absolute value, then $|\# G|=1$ thus for every $x \in V_{F},|\tilde{f}(x)|=\frac{1}{|\# G|}\left|\sum_{g \in G} g(x)\right|=$ $\left|\sum_{g \in G} g(x)\right| \leq \max _{g \in G}|g(x)| \leq N(x)$ whence $|\tilde{f}| \leq N_{\uparrow V_{F}}$.

(2) If the characteristic of the field $\mathbb{F}$ is zero, then $\mathbb{F}$ extends the field $\mathbb{Q}$ of rational numbers. Since $|\cdot|_{i \mathbb{Q}}$ is non-trivial, Ostrowski's theorem implies that the absolute value induced by $|$.$| on \mathbb{Q}$ is equivalent to the $p$-adic absolute value for some prime number $p$. With $\mathbf{M C}(p)$, let $\Phi_{p}$ be a mapping associating to each non-empty subset $X$ of $\mathbb{F}^{E}$ a finite subset $G$ of $X$ such that $p$ does not divide $\# G$. Let $G:=\Phi_{p}(\mathcal{G})$ : then $G$ is a finite subset of $\mathcal{G}$ such that $p$ does not divide $\# G$. Let $n:=\# G$. Then $|n|=1$ : in the first subcase, $n \in \mathbb{F}_{p} \backslash\{0\} \subseteq \mathbb{F}$ thus $|n|=1$; in the second subcase, $|n|_{p}=1$ because $p$ does not divide $n$, thus $|n|=1$.

Now we consider the linear form $\tilde{f}:=\frac{1}{n} \sum_{g \in G} g$ : this linear form extends $f$, and for every $x \in V_{F},|\tilde{f}(x)|=\frac{1}{|n|}\left|\sum_{g \in G} g(x)\right|=\left|\sum_{g \in G} g(x)\right| \leq$ $\max _{g \in G}(|g(x)|) \leq N(x)$, whence $|\tilde{f}| \leq N_{\uparrow V_{F}}$.

Corollary 2. (1) MC implies $\mathbf{H B}$ and $\mathbf{I}_{(\mathbb{F},|.|)}$ for every spherically complete ultrametric field $(\mathbb{F},||$.$) with zero characteristic such$ that $|\cdot|_{\mathbb{Q}}$ is trivial. 
(2) For every prime number $p, \mathbf{M C}(p)$ implies $\mathbf{I}_{(\mathbb{F},|.|)}$ for every spherically complete ultrametric field with characteristic $p$ or with zero characteristic such that $|.|_{\mathbb{Q}}$ is equivalent with the absolute value $|.|_{p}$ of $\mathbb{Q}$.

Proof. Proof by transfinite recursion using Theorem 1.

Remark 9. The implication $\mathbf{M C} \Rightarrow \mathbf{H B}$ was already proved in 14 (MC implies Rado's Lemma which implies HB). The implication $\mathbf{M C} \Rightarrow \mathbf{I}_{(\mathbb{F},|.|)}$ was proved for every spherically complete ultrametric field $(\mathbb{F},||$.$) with null characteristic such that |.|_{\wedge \mathbb{Q}}$ is trivial (see [15, Proposition 4.4]).

It follows that the "full Ingleton theorem" follows from $\forall^{\mathrm{PRIME}} p \mathbf{M C}(p)$ in set theory ZFA, thus in ZFA, the full Ingleton statement does not imply AC.

\subsection{Some questions.}

(1) Are there links in set-theory without choice between the statements $\mathbf{I}_{\mathbb{F}}$ obtained for various spherically complete ultrametric valued fields $\mathbb{F}$ ?

(2) Given a prime number $p$ :

-Denoting by $\mathbb{F}_{p}$ the finite field with $p$ elements, does $\mathbf{I}_{\mathbb{F}_{p}}$ implies $\mathbf{I}_{\mathbb{F}}$ for every spherically complete ultrametric valued field with characteristic $p$ ?

-Does $\mathbf{I}_{\mathbb{Q}_{p}}$ imply $\mathbf{I}_{(\mathbb{F},|.|)}$ for every spherically complete ultrametric valued field $(\mathbb{F},||$.$) with null characteristic such that \left.\left.\right|_{\mid}\right|_{\mathbb{Q}}$ is equivalent to $|\cdot|_{p}$ ?

(3) Does the conjunction of the statements $\mathbf{I}_{\mathbb{Q}_{p}}$ for $p$ prime number imply $\mathbf{I}_{\left(\mathbb{Q},\left.|\cdot|\right|_{\text {triv }}\right)}$ or $\mathbf{H B}$ ?

(4) Given two different prime numbers $p$ and $q$, are the statements $\mathbf{I}_{\mathbb{Q}_{p}}$ and $\mathbf{I}_{\mathbb{Q}_{q}}$ equivalent?

Remark 10. For each ultrametric spherically complete valued field $(\mathbb{F},||$.$) ,$ the statement $\mathbf{I}_{(\mathbb{F},|.|)}$ is equivalent to the following one (see [15]):

"For every vector subspace $F$ of an ultrametric semi-normed $\mathbb{F}$-vector space $(E, N)$, there exists an isometric linear extender $T: B L(F, \mathbb{F}) \rightarrow$ $B L(E, \mathbb{F})$." Here, given a vector subspace $V$ of $E, B L(V, \mathbb{F})$ denotes the set of linear bounded mappings from $V$ to $\mathbb{F}$.

\section{In ZFA, MC implies the Krein-Milman statement KM}

4.1. Facets of subsets of a real vector space. Given a real vector space $E$, and two elements $a, b \in E$, we denote by $[a, b]$ the segment subset $\{t . a+(1-t) . b: t \in[0,1]\}$ of $E$; if $a=b$, then $[a, b]$ is a singleton, 
else the segment $[a, b]$ is infinite (it is equipotent with $[0,1]$ ) and we denote by $] a, b[$ the strict segment $] a, b[:=\{t . a+(1-t) . b: t \in] 0,1[\}$. A subset $C$ of $E$ is convex if for every $a, b \in C,[a, b] \subseteq C$. In particular, every segment is convex. Say that an element $e$ of a convex subset $C$ of $E$ is an extreme point of $C$ is $C \backslash\{e\}$ is convex: this means that for every distinct elements $a, b$ of $C$, if $e \in[a, b]$ then $e=a=b$. Say that a subset $F$ of a subset $X$ of $E$ is a facet of $X$ if $F$ is nonempty, and if for every distinct elements $a, b$ in $X$, if $[a, b] \subseteq X$ and $] a, b[$ meets $F$ then $[a, b] \subseteq F$.

Remark 11. A facet of a convex subset $X$ may be not convex: for example $\{0,1\}$ is a non-convex facet of the subset $[0,1]$ of the onedimensional vector space $\mathbb{R}$.

Remark 12. Given an element $e$ of a convex subset $C$ of $E$, $e$ is an extreme point of $C$ if and only if $\{e\}$ is a facet of $C$.

Remark 13. Given a subset $X$ of a real vector space $E$,

(1) Every facet of a facet of $X$ is a facet of $X$;

(2) A nonempty set which is an intersection of facets of $X$ is a facet of $X$;

(3) A union of a nonempty set of facets of $X$ is a facet of $X$;

(4) if $X$ is nonempty then $X$ is a facet of $X$.

Given a subset $X$ of a real vector space $E$, a mapping $f: X \rightarrow$ $\mathbb{R}$ is said to be convex if for every $a, b \in X$ and every $s \in[0,1]$, if $s . a+(1-s) \cdot b \in X$ then $f(s . a+(1-s) \cdot b) \leq s f(a)+(1-s) f(b)$. The mapping $f: X \rightarrow \mathbb{R}$ is said to be concave if $-f$ is convex.

Lemma 3. Given a real vector space $E$, a subset $X$ of $E$ and a convex mapping $f: X \rightarrow \mathbb{R}$ which is upper bounded and attains its least upper bound $M$, then the subset $F:=\{x \in X: f(x)=M\}$ is a facet of $X$.

Proof. Assume that there are two distinct elements $a, b \in X$ such that ]$a, b[$ meets $F$ and $[a, b] \subseteq X$. Let us show that $[a, b] \subseteq F$. Since $] a, b[$ meets $F$, let $u \in] a, b[$ such that $f(u)=M$ : then there exists some real number $s$ such that $0<s<1$ and $u=s . a+(1-s) . b$. By convexity of $f, M=f(s . a+(1-s) . b) \leq s f(a)+(1-s) f(b)$; if $f(a)<M$ or $f(b)<M$, then $s f(a)+(1-s) f(b)<M$ which is contradictory. Thus $f(a)=f(b)=M$. Now if $x \in] a, b[$ and $x \neq u$, then $u \in] a, x[$ or $u \in] x, b[$ whence $f(x)=M$ by convexity of $f$. Thus $[a, b] \subseteq F$.

Given a set $X$, a set $\mathcal{C}$ of subsets of $X$ is said to satisfy the finite intersection property if every finite nonempty subset of $\mathcal{C}$ has a nonempty intersection. A subset $X$ of a real linear topological vector space $E$ is 
convex-compact (see [13, p. 135]) if for every family $\mathcal{C}$ of closed convex subsets of $E$ such that $\{C \cap X: C \in \mathcal{C}\}$ satisfies the finite intersection property, $\cap \mathcal{C} \cap X$ is nonempty.

Lemma 4. Given a real topological vector space $E$, a convex-compact subset $X$ of $E$ and a continuous concave mapping $f: E \rightarrow \mathbb{R}$ which is not constant on $X$, then $f$ is upper bounded on $X$ and attains its supremum $M$ on $X$; moreover, if $f$ is linear, then the closed subset $\{x \in X: f(x)=M\}$ of $X$ is a facet of $X$.

Proof. For every real number $\lambda \in f[X]$, let $C_{\lambda}:=\{x \in E: f(x) \geq \lambda\}$; since $f$ is continuous and concave, $C_{\lambda}$ is a closed convex subset of $E$; moreover, since $\lambda \in f[X], X \cap C_{\lambda} \neq \varnothing$. Let $\mathcal{C}:=\left\{C_{\lambda}: \lambda \in f[X]\right\}$. Then $\{C \cap X: C \in \mathcal{C}\}$ satisfies the finite intersection property, thus $F:=\cap \mathcal{C} \cap X=\{x \in X: f(x)=M\}$ is nonempty; let $x_{0} \in F$ : then $M:=f\left(x_{0}\right)=\sup _{X} f$. Moreover, if $f$ is linear, then $f$ is convex, thus Lemma 3 implies that the closed subset $F$ of $X$ is a facet of $X$.

Remark 14. The statement $\mathbf{H B}$ is equivalent to the following statement:

Given a real Hausdorff locally convex topological vector space E, for every non null element $x \in E$, there exists a continuous linear functional $f: E \rightarrow \mathbb{R}$ such that $f(x)=1$.

Proof. $\Rightarrow$ Given some real Hausdorff locally convex topological vector space $E$ and some non-null element $x$ of $E$, consider some convex open neighbourhood $V$ of $0_{E}$ such that $x \notin V$. Then, Theorem 2 of [3] implies the existence of a linear functional $f: E \rightarrow \mathbb{R}$ such that $\sup _{V} f<f(x)$. Since $f: E \rightarrow \mathbb{R}$ is linear and bounded on a neighbourhood of $0_{E}, f$ is continuous. For the converse statement, see [4, Lemma 5].

Remark 15. It follows that given a subset $K$ of a real locally convex Hausdorff topological vector space $E$, if $K$ is convex-compact in $E$, then $\mathbf{H B}$ implies that closed minimal facets of $K$ are singletons of $K$.

Proof. Given a closed facet $F$ of $K$ which is not a singleton, consider with HB some continuous linear functional $f: E \rightarrow \mathbb{R}$ which is not constant on $F$; then, the closed subset $F$ of $K$ is convex-compact, thus $f$ attains its upper bound $M$ on $F$; using Lemma 4, the set $\{x \in F$ : $f(x)=M\}$ is a proper facet of the set $F$, thus the facet $F$ of $K$ is not minimal.

\subsection{Trees of subsets of a set.}


4.2.1. Trees. A tree is a partially ordered set $(T, \preceq)$ with a smallest element $r$ (called the root of the tree) such that for every $x \in T$, the interval $\{y \in T: y \preceq x\}$ is well ordered. Thus a tree is a well founded partially ordered set $(T, \preceq)$ such that for every $x \in T$, the interval $\{y \in T: y \preceq x\}$ is linearly ordered.

Remark 16. Given an element $x$ of a tree $(T, \preceq)$, the set $\{y \in T: y \preceq x\}$ is the smallest chain of $T$ containing $x$ and the root of $T$.

Given an element $x$ of a tree $(T, \preceq)$, we denote by $x^{+}$the set of elements $y \in T$ which cover $x$ i.e. such that $x \prec y$ (where $\prec$ is the strict-order associated to $\preceq$ ) and the interval $] x, y[$ is empty; elements of $x^{+}$are called successors of $x$. Given elements $x, y \in T$, if $y$ is a successor of $x$, then $x$ is said to be a predecessor of $y$. Every element of $X$ has at most one predecessor in the tree $T$. A leaf of the tree $(T, \preceq)$ is a maximal element of the $\operatorname{poset}(T, \preceq)$. Thus an element $x$ of a tree $T$ is a leaf of $T$ if $x$ has no successor in $T$. A branch of the tree $(T, \preceq)$ is a maximal chain of the poset $(T, \preceq)$ : every branch has a first element which is the root of the tree. Leaves of a tree correspond to greatest elements of branches of this tree. A subtree of a tree $(T, \preceq)$ is a nonempty initial section of the poset $(T, \preceq)$ i.e. a subset of $T$ containing the root of $T$ and such that for each $x \in S,\{y \in T: y \preceq x\} \subseteq S$. Of course, each subtree of a tree $(T, \preceq)$ is a tree for the order induced by $\preceq$ on the subtree.

Remark 17. In ZFA, the Axiom of Choice implies that every tree has a branch. The converse implication is also true since the existence of a branch in every tree implies the statement $\forall \kappa \mathbf{D C} \mathbf{C}_{\kappa}$ (form $1 \mathrm{~F}$ in $[7$, p. 12]) which in turn implies $\mathbf{A C}$ ([10, Th. 8.2. p. 121]).

Given a tree $(T, \preceq)$ with root $r$, since the $\operatorname{poset}(T, \preceq)$ is well-founded, we may consider the "rank function" rk associating to each $x \in T$ the ordinal such that $\operatorname{rk}(r)=0$ and, for each $x \in T \backslash\{r\}, \operatorname{rk}(x)=$ $\sup \{\operatorname{rk}(y)+1: y \prec x\}$. An element $y \in T$ has a predecessor if and only if the ordinal $\operatorname{rk}(y)$ is a successor ordinal. Denoting by On the collection of ordinals, we consider the family $\left(L_{\alpha}\right)_{\alpha \in \text { On }}$ of level sets of the tree $T$, where for each ordinal $\alpha, L_{\alpha}$ is the set of elements of $T$ with rank $\alpha$. Thus $L_{0}=\{r\}$ where $r$ is the root of the tree $T$. For each ordinal $\alpha$, we denote by $T_{\alpha}$ the set $\{x \in T: \operatorname{rk}(x)<\alpha\}$. Thus $T_{0}=\varnothing$, and for each ordinal $\alpha \geq 1, T_{\alpha}$ is a subtree of $T$. Moreover, for each ordinal $\alpha, T_{\alpha+1}=T_{\alpha} \cup L_{\alpha}$, and for each limit ordinal $\alpha>0$, $T_{\alpha}=\cup_{\beta \in \alpha} T_{\beta}$.

Notice that for every ordinal $\alpha$, if $L_{\alpha}=\varnothing$, then for every $\beta \geq \alpha$, $L_{\beta}=\varnothing$ and $T_{\beta}=T_{\alpha}$. Since $T$ is a set, the axiom schema of replacement 
implies that there exists an ordinal $\alpha$ such that $T_{\alpha+1}=T_{\alpha}$ (whence $\left.L_{\alpha}=\varnothing\right)$. The first ordinal $\alpha$ such that $L_{\alpha}$ is empty is called the rank of the tree $T$.

4.2.2. Trees of subsets of a set $X$. Given a set $X$, a tree of subsets of $X$ is a subset $T$ of the poset $(\mathcal{P}(X) \backslash\{\varnothing\}), \supseteq)$, with smallest element $X$, such that the induced poset on $T$ is a tree, and such that for every $x \in T$, successors of $x$ are pairwise disjoint subsets of $X$. It follows that for every $x, y \in T$, if $x$ and $y$ are not comparable for the inclusion relation then $x \cap y=\varnothing$.

Remark 18. Given a tree $T$ of subsets of a set $X$ with rank $\alpha$, and denoting for each $\beta \in \alpha$ by $L_{\beta}$ the level set $\{x \in T: \operatorname{rk}(x)=\beta\}$, and by $F_{\beta}$ the subset $\cup L_{\beta}$ of $X$, then the family $\left(F_{\beta}\right)_{\beta \in \alpha}$ is descending i.e. for every $s, t \in \alpha$ such that $s \in t, F_{t} \subseteq F_{s}$.

Proof. Given $b \in F_{t}$, there exists $y \in L_{t}$ such that $b \in y$. Let $x$ be the element of $L_{s}$ such that $x \preceq y$; then $y \subseteq x$ thus $b \in x \subseteq F_{s}$.

Lemma 5. If $T$ is a tree of nonempty closed subsets of a compact topological space $X$, and if each level of $T$ is finite, then $T$ has at least one branch.

Proof. Let $\alpha$ be the rank of the tree $T$. For each $\beta<\alpha$, let $F_{\beta}$ be the (nonempty) closed subset $\cup L_{\beta}$. Then the family $\left(F_{\beta}\right)_{\beta \in \alpha}$ is decreasing thus the set $\cap_{\beta<\alpha} F_{\beta}$ is nonempty: let $x \in \cap_{\beta<\alpha} F_{\beta}$; let $b:=\{F \in T$ : $x \in F\}$; since elements of $T$ are pairwise disjoint, $b$ is a branch of $T$.

4.2.3. Extending branches of a tree of subsets. Given a set $X$ and a tree $T$ of subsets of $X$, given a branch $b$ of $T$ with no leaf, if $\cap b \neq \varnothing$, and if $F$ is a set of pairwise disjoint nonempty subsets of $\cap b$, then $b \cup F$ is a tree of subsets of $X$ : we call it the tree obtained by extending the branch $b$ with successors in $F$. Given a nonempty set $B$ of branches of $T$, if for each $b \in B, F_{b}$ is a nonempty set of nonempty pairwise disjoint subsets of $\cap b$, then $\cup B \cup \cup_{b \in B} F_{b}$ is a tree of subsets of $X$ which is called the tree obtained from the subtree $\cup B$ by extending each $b \in B$ by the set of successors $F_{b}$.

4.2.4. Dynasties of trees of subsets. A dynasty of trees of subsets of $X$ is a collection $\left(T_{\alpha}\right)_{\alpha \in \text { On }}$ of trees of subsets of $X$, such for each $\alpha \in$ On, there exists a set $B$ of branches of the tree $S:=\cup_{\beta<\alpha} T_{\beta}$ such that either $T_{\alpha}$ is the subtree $\cup B$ of $S$, or $T_{\alpha}$ is obtained from the tree $\cup B$ by extending each branch $b \in B$ by a nonempty set of successors. 


\subsection{The axiom of "Multiple Choices" MC implies the Krein- Milman axiom.}

Lemma 6. Given a real vector space $E$ and a nonempty subset $X$ of $E$, there is a mapping associating to each nonempty finite set $F$ of convex facets of $X$ a nonempty finite set $G$ of pairwise disjoint convex facets of $X$ such that for each $y \in G$, there exists $x \in F$ such that $y \subseteq x$.

Proof. Let $\mathcal{F}$ the set of convex facets of $X$. Given a nonempty finite subset $F$ of $\mathcal{F}$, let $F_{1}$ be the set of maximal subsets $J$ of $F$ such that $\cap J$ is nonempty; then $G:=\left\{\cap J: J \in F_{1}\right\}$ is a set of convex facets of $X$ such that for each $C \in G$, there exists $C^{\prime} \in F$ satisfying $C \subseteq C^{\prime}$; elements of $G$ are pairwise disjoint because elements of $F_{1}$ are maximal subsets of $F$ with nonempty intersection.

\section{Theorem 2. In ZFA, MC implies KM.}

Proof. Let $E$ be a real locally convex Hausdorff topological vector space and let $K$ be a nonempty convex compact subset of $E$. Using MC, let $\Phi$ be a mapping associating to each nonempty set $A$ of subsets of $K$ a nonempty finite subset of $A$. We shall build by recursion a dynasty $\left(T_{\alpha}\right)_{\alpha \in \text { On }}$ of trees of closed convex facets of $K$, such that for each $\alpha \in \mathrm{On}$, the tree $T_{\alpha}$ has finite levels. The tree $T_{0}$ is the singleton $\{K\}$ (here $K$ is a facet of $K$ ). Given an ordinal $\alpha>0$ such that the family $\left(T_{\beta}\right)_{\beta \in \alpha}$ has been defined, and denoting by $S$ the tree $\cup_{\beta<\alpha} T_{\beta}$, Lemma 5 implies that the set of branches of the tree $S$ is nonempty (because $S$ is a tree of closed subsets of a compact space and each level of $S$ is finite). Using $\Phi$, we consider a nonempty finite set $B$ of branches of $S$, and we distinguish two cases according to whether there exists $b \in B$ such that $\cap b$ is a minimal facet of $K$ or not. In the first case and if there exists $b \in B$ such that $b$ has a leaf, then $T_{\alpha}:=\cup\{b \in B$ : $b$ has a leaf in $S\}$. If no branch $b \in B$ has a leaf, then, for each $b \in B$, $F_{b}:=\cap b$ is a minimal facet of $K$ and we define $T_{\alpha}=B\left[\left(F_{b}\right)_{b \in B}\right]$. In the second case, we consider, with $\Phi$ and Lemma 6 , for each $b \in B$, a nonempty finite set $F_{b}$ of pairwise disjoint convex proper facets of $\cap b$, and we define the tree $T_{\alpha}=B\left[\left(F_{b}\right)_{b \in B}\right]$ obtained from the subtree $\cup B$ by extending each $b \in B$ by the set $F_{b}$ of successors. Using the axiom schema of replacement, there exists an ordinal $\alpha$ such that $T_{\alpha}=T_{\alpha+1}$, thus $T_{\alpha}$ has a leaf which is a closed minimal convex facet of $K$ : this facet corresponds to an extreme point of $K$.

Corollary 3. In ZFA, MC implies that every compact convex subset of a real Hausdorff locally convex topological vector space is the closed convex hull of the set of its extreme points. 
Proof. Given a nonempty compact convex subset $K$ of a real Hausdorff locally convex topological vector space $E, \mathbf{M C}$ implies that the set $X$ of extreme points of $K$ is nonempty (see Theorem 2); then using HB (which is a consequence of $\mathbf{M C}$ ), it follows that $K$ is the closed convex hull of $X$.

Remark 19. Consider the following statement VKM: "Every nonempty convex-compact convex subset of a real locally convex topological vector space has an extreme point." (form 286 in [7]). It is known that HB + VKM implies AC ([2]), thus $\forall^{\mathrm{PRIME}} p \mathbf{M C}_{p}$ does not imply VKM. Also notice that VKM implies $\forall \kappa \mathbf{A C}^{\kappa}$ (see [4]). However, it is an open question whether VKM implies AC.

\section{REFERENCES}

[1] Y. Amice. Les nombres p-adiques. Presses Universitaires de France, Paris, 1975. Préface de Ch. Pisot, Collection SUP: Le Mathématicien, No. 14.

[2] J. Bell and D. Fremlin. A geometric form of the axiom of choice. Fundam. Math., 77:167-170, 1972.

[3] J. Dodu and M. Morillon. The Hahn-Banach property and the Axiom of Choice. Math. Log. Q., 45(3):299-314, 1999.

[4] J. Fossy and M. Morillon. The Baire category property and some notions of compactness. J. Lond. Math. Soc., II. Ser., 57(1):1-19, 1998.

[5] F. Q. Gouvêa. p-adic numbers. Universitext. Springer-Verlag, Berlin, second edition, 1997. An introduction.

[6] W. Hodges. Läuchli's algebraic closure of Q. Math. Proc. Cambridge Philos. Soc., 79(2):289-297, 1976.

[7] P. Howard and J. E. Rubin. Consequences of the Axiom of Choice, volume 59. American Mathematical Society, Providence, RI, 1998.

[8] P. E. Howard. Rado's selection lemma does not imply the Boolean prime ideal theorem. Z. Math. Logik Grundlag. Math., 30(2):129-132, 1984.

[9] A. W. Ingleton. The Hahn-Banach theorem for non-Archimedean valued fields. Proc. Cambridge Philos. Soc., 48:41-45, 1952.

[10] T. J. Jech. The Axiom of Choice. North-Holland Publishing Co., Amsterdam, 1973.

[11] H. Läuchli. Auswahlaxiom in der Algebra. Comment. Math. Helv., 37:1-18, $1962 / 1963$.

[12] A. Levy. Axioms of multiple choice. Fund. Math., 50:475-483, 1962.

[13] W. A. J. Luxemburg. Reduced powers of the real number system and equivalents of the Hahn-Banach extension theorem. In Applications of Model Theory to Algebra, Analysis, and Probability (Internat. Sympos., Pasadena, Calif., 1967), pages 123-137. Holt, Rinehart and Winston, New York, 1969.

[14] M. Morillon. Some consequences of Rado's selection lemma. Arch. Math. Logic, 51(7-8):739-749, 2012.

[15] M. Morillon. Linear extenders and the axiom of choice. Comment. Math. Univ. Carolin., 58(4):419-434, 2017. 
[16] J. Pawlikowski. The Hahn-Banach theorem implies the Banach-Tarski paradox. Fund. Math., 138(1):21-22, 1991.

[17] D. Pincus. Independence of the prime ideal theorem from the Hahn Banach theorem. Bull. Amer. Math. Soc., 78:766-770, 1972.

[18] A. M. Robert. A course in p-adic analysis, volume 198 of Graduate Texts in Mathematics. Springer-Verlag, New York, 2000.

[19] A. C. M. van Rooij. Non-Archimedean functional analysis, volume 51 of Monographs and Textbooks in Pure and Applied Math. Marcel Dekker, Inc., New York, 1978.

[20] A. C. M. van Rooij. The axiom of choice in $p$-adic functional analysis. In $p$-adic functional analysis (Laredo, 1990), volume 137 of Lecture Notes in Pure and Appl. Math., pages 151-156. Dekker, New York, 1992.

Laboratoire d'Informatique et Mathématiques, Parc Technologique Universitaire, BÂtiment 2, 2 RUe Joseph Wetzell, 97490 Sainte Clotilde E-mail address: Marianne.Morillon@univ-reunion.fr 